\title{
THE CRANIAL HYPEROSTOSES PRODUCED BY MENINGEAL ENDOTHELIOMAS *
}

\author{
HARVEY CUSHING, M.D. \\ BOSTON
}

This communication will be chiefly devoted to but one of the many interesting features of the endotheliomas, namely, the hyperplasia of the adjacent bone. That the relationship between the meningeal tumor and the hyperostosis is not generally understood was brought out at a recent meeting of the Society of Neurological Surgeons held in Philadelphia. At that time Professor Spiller exhibited many interesting specimens from his extensive collection of brain tumors. In showing some examples of endotheliomas he stated that these tumors were sometimes associated with an overlying cranial hyperostosis which he considered to be the cause of the subjacent growth. This opinion was so contrary to that which has long been held and taught in the writer's clinic, that this occasion is taken to present some data bearing on this particular aspect of these lesions.

It is quite probable that the true character of the bony tumor has often been overlooked because the necessity of decalcification has deterred observers from making a careful histologic study of the adjacent portion of the skull as well as of the tumor proper. This, I must admit, applies to some of the early examples of hyperostosis cranei accompanying endotheliomas in my Johns Hopkins series. My attention was first drawn to the true nature of the bony tumor by an experience which may be briefly stated.

The patient came under my care in February, 1910, giving the following history: Twelve years before he had received a sharp blow on the vertex of the head approximately at the rolandic point. This point remained somewhat tender, and in the course of time a local prominence of the skull, attributed to an epicranial exostosis, became apparent. Occasional attacks of focal epilepsy involving the left foot and leg subsequently occurred, and under the assumption that these attacks were produced by cortical irritation, a surgeon in 1905 trephined and removed the small area of thickened bone. The underlying dura which was exposed was thought to be normal, and the membrane was not opened. The thickened disk of bone which had been removed showed tumor on microscopic examination. It was classified by a pathologist as a primary psammosarcoma of the diploe.

For the next five years the patient's jacksonian attacks continued unabated, with increasing disability and spasticity of the affected foot. There was no local recurrence of the tumor at the site of the bone defect, and in the absence

\footnotetext{
* From the Surgical Clinic of the Peter Bent Brigham Hospital.
} 
of the usual manifestations of pressure (headache, choked disk, etc.) an intracranial tumor was not suspected by any of the several neurologists and surgeons who were subsequently consulted.

By the time of his admission to the hospital in 1910 the left arm had begun to be involved in the spasticity and an osteoplastic exploration was decided on. This disclosed a large potato-like psammo-endothelioma which had greatly deformed the right hemisphere. The tumor, which weighed $198 \mathrm{gm}$., was successfully enucleated. Its meningeal attachment was situated alongside the sinus sagittalis, directly under the small bone defect made at the original operation.

Though the dural attachment of the tumor at the sinus was not removed and was unquestionably infiltrated by tumor, there has been no evidence of continued growth though twelve years have elapsed.

Since this experience, areas of cranial thickening, whether disclosed by palpation or by roentgen ray, have always been looked on with the suspicion that they might overlie a meningeal endothelioma. It, however, was not appreciated for some time that these tumors in their intradural aspects might be of two distinct types: (1) massive tumors with a relatively small area of meningeal attachment, and (2) tumors en plaque which are but slightly elevated though they may involve a considerable area of dura. Though the former may in a certain percentage of the cases be accompanied by an overlying hyperostosis, the bony thickening is apt to be much more pronounced in the case of the flat tumors. As a matter of fact, were it not for the hyperostosis and its consequences, many of these meningeal tumors would pass unrecognized, for they may give no intracranial symptoms of tumor either general or localizing. Indeed, the massive, potato-like endotheliomas may sometimes attain very large dimensions before general pressure symptoms, such as choked disk or headaches, are manifested. This was true of the patient with the 198-gram tumor whose case has just been mentioned.

The endotheliomas have their definite seats of predilection, a full discussion of which must await a subsequent and more extensive report. It will suffice, for the present, to say that the tumors which are parasagittal in origin, and those which arise from the temporofrontal meninges adjacent to the sylvian cleft seem to be accompanied by a hyperostosis cranei more often than those in other situations.

For purposes of this report, a single comparatively recent example of one of these temporal lesions has been chosen. The region is one in which endotheliomas frequently arise, and when they are associated with marked hyperostosis, as in this particular case, exophthalmos is almost invariably produced.

REPORT OF A CASE

Left temporosphenoidal endothelioma en plaque, with invasion and thickening of overlying bonc and extracranial involvement of temporal muscle. 
April 18, 1921.-Admission of Mrs. Mary D., aged 39, referred by Dr. Byrnes of Springfield, Mass., with a bony tumor of the orbit, producing exophthalmos.

Past History.-No apparent bearing on present trouble. Excellent general health. No recorded trauma. Occasional headaches since childhood; none for past year. Married at 27 years: six pregnancies with only two children surviving. Four of them were stillborn at seven months: attributed to renal complications. Last pregnancy was terminated June, 1920, because of eclampsia and convulsions: placenta praevia.

Present Complaint.-For possibly ten years the left eye has been somewhat more prominent than the other. No significance was attached to this, even when in September of 1919 the eyelids on this side became somewhat edematous. As she had some albuminuria and her ankles were apt to be swollen. the puffiness of the eyclids, though unilateral and persistent, was discounted. In Felru-

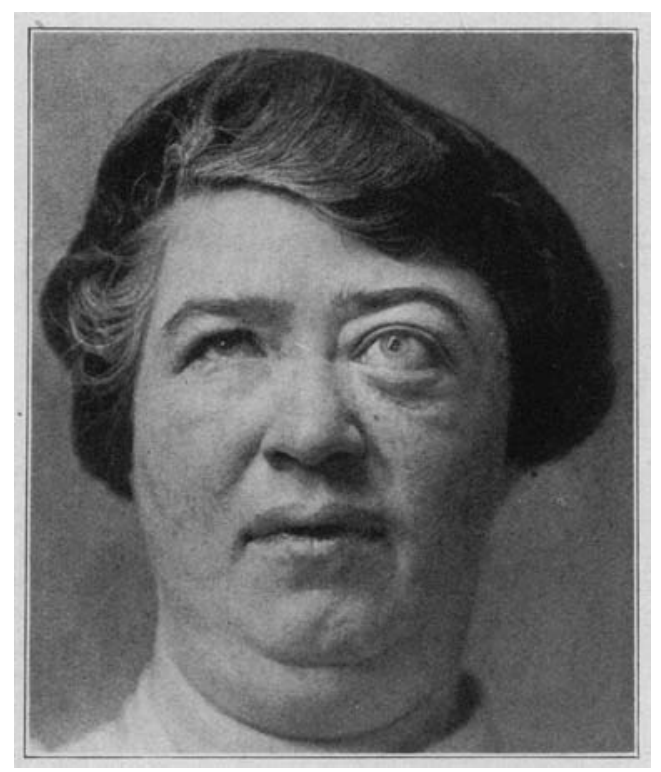

Fig. 1.-Appearance on adnission, showing exophthalmos. The bony protrusion is evident, though partly concealed by her hair.

ary, 1920, she first observed a swelling in the anterior part of the left temporal fossa. It was painless, hard, not sensitive to pressure. Her physician at the time evidently regarded the condition as syphilitic and prescribed the usual drugs, but she did not persist in their use.

On the termination of her pregnancy in June she first noticed some slight loss of visual acuity in the left eye.

For the next six months there was a slow but steady enlargement of the aforementioned bony tumor of the temporal fossa. accompanied by an increas. ing prominence of the eye and edema of the lids. For the three months before admission the symptoms had developed more rapidly.

Physical Examination.-This was essentialiy negative except for the obvious local lesion, namely, a smooth, hard tumor, slightly tender on pressure, filling out the left temporal fossa and obliterating such bony landmarks as the external 
angular process and zygoma. Associated with the growth was a moderate unilateral exophthalmos with edema of the eyelids (Fig. 1). The exophthalmos was without bruit or pulsation, though over the bony tumor the extracranial vessels were noticeably dilated. The patient was markedly mopic and a slight hyperemia, particularly of the left optic disk, was present. The visual fields were normal. There was slight weakness of the left abducens.

The situation of the growth was obscurely shown in a series of stereoscopic roentgen-ray plates. These (Fig. 2) indicated an area of increased density in the region of the squamous wing of the temporal bone, the sphenoid, and left side of the orbit.

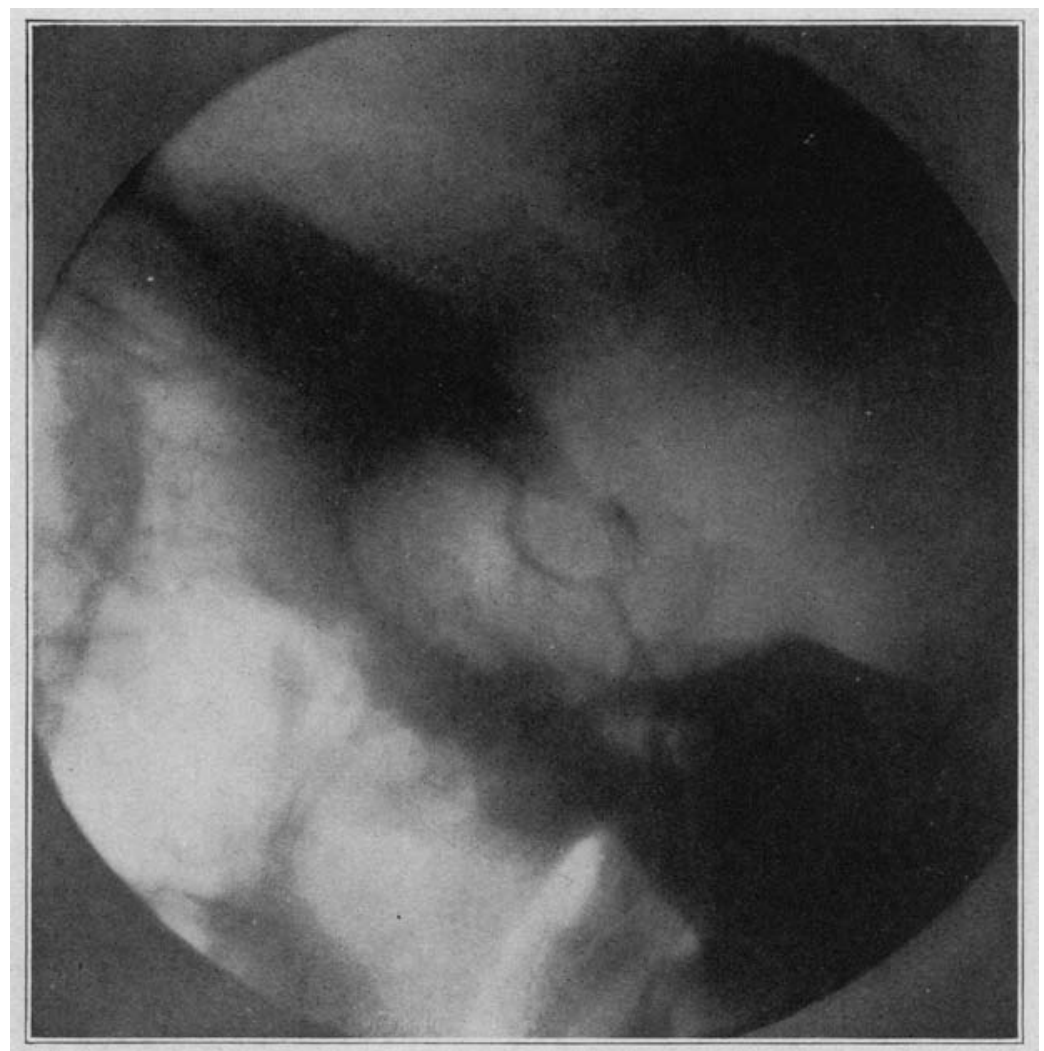

Fig. 2.-Lateral roentgenogram of sellar region showing area of increased density

The neurologic examination was negative in all respects. The llassermann reaction was negative. Urine showed a trace of albumin, but functional renal tests revealed no such degree of dysfunction as to contraindicate operation.

May 4, 1921: Operation. Extirpation of hyperostosis togcther with underlying dura and its subjacent fat tumor.-A curvilinear incision (Fig. 4) was made with its base between the external angular process and the tragus. On reflecting the scalp and temporal muscle so as to expose the bony lesion, it was found that the lower layer of muscle fibers were infiltrated by tumor over the most prominent part of the bony protuberance. The skull at this point was 
considerably roughened, and the muscle was adherent to it. This small area of obvious involvement of extracranial tissues was excised and an immediate frozen section showed endothelioma.

The area of hyperostosis, which was difficult of access, was then attacked by making a circle of perforations at its periphery. These perforations, made by using a succession of motor-driven burrs of graduated size, were carried down through the dense bone to the dura. It would probably have been easier to have "burred" away the entire hyperostosis in this fashion, but it was desired to preserve an intact portion of the thickened bone for subsequent study.

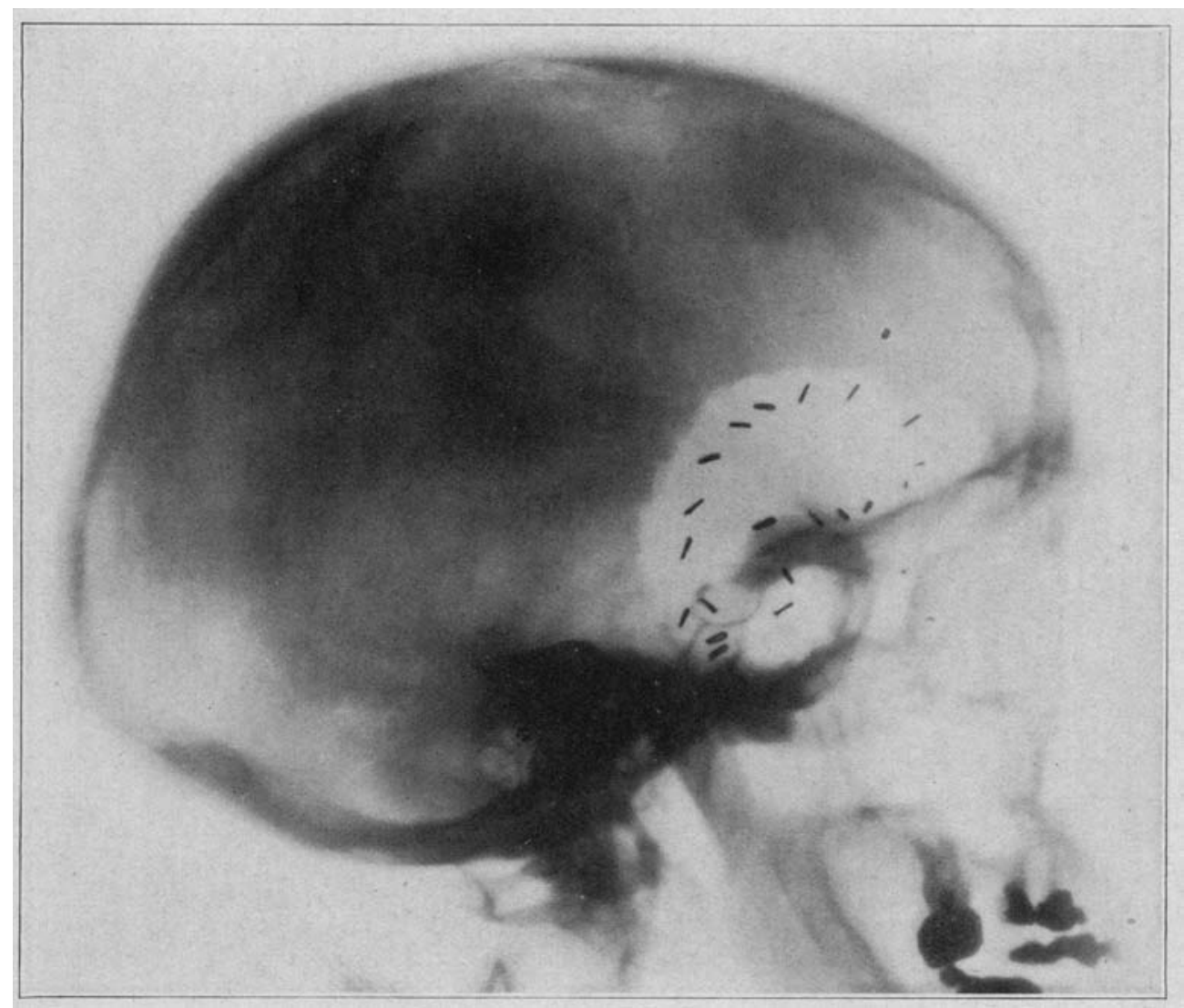

Fig. 3.-Print of postoperative radiogram showing extent of bony removal and situation of clips outlining area of excised dura.

The chief difficulty lay in the complete removal of the hyperostosis at its anterior and lower periphery. In this situation it was necessary to carry the perforations obliquely through solid bone which proved to be 3 to $4 \mathrm{~cm}$. in thickness, before the orbital cavity was reached. The remaining central and posterior mass of bone (Figs. 9-11) was then broken out in a single piece. $W$ ith the view thus given it was possible, with the further use of burrs and rongeurs, to enlarge the opening until all the thickened bone so far as could be told, was removed. In this procedure the entire outer side of the orbital contents, which had evidently been considerably encroached on by the growth, 


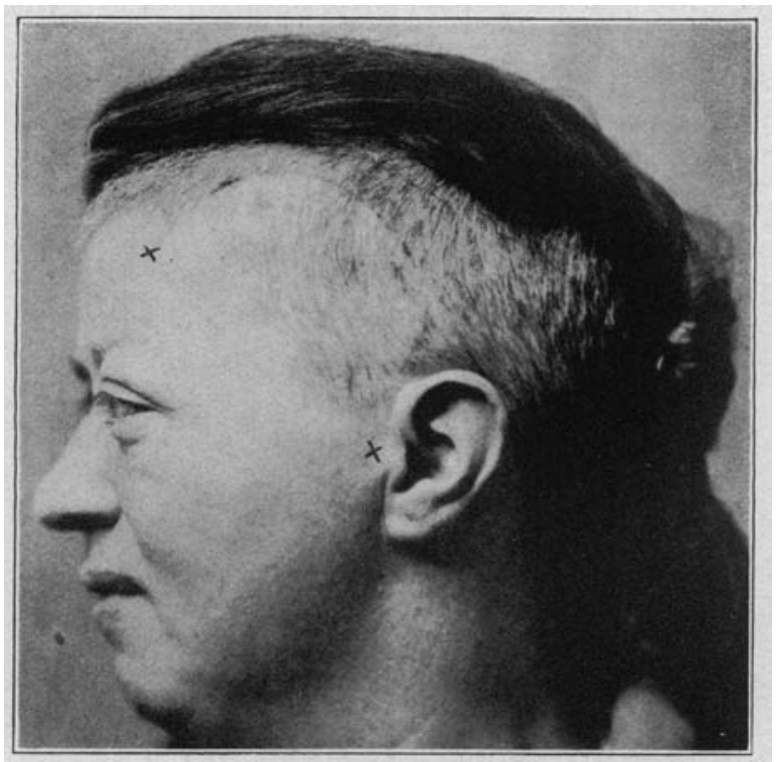

Fig. 4.-Twelve days after operation, to show the field and situation of the curved incision, its limits indicated by crosses.

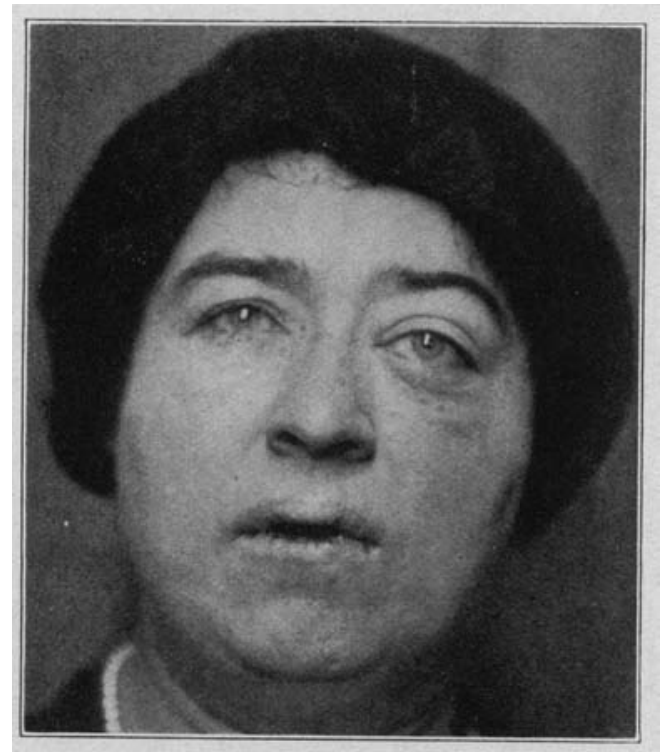

Fig. 5.-Condition ten months after operation (for comparison with Fig. 1 ). 
was fully exposed. Removal of the sphenoid wing was carried inward to the region of the left anterior clinoids in the depths of the sylvian groove.

At the bottom of the bone defect thus made, roughened dura was exposed covering practically the entire tip of the temporal lobe. There was no certainty as to what underlay this thickened dura, whether an endothelioma of the plaque variety or a rounded tumor. The membrane was opened at the upper margin of the bone defect where it was evidently normal in appearance and the edge of a flat endothelioma was seen. The incision in the membrane was then carried around between normal dura and the edge of the tumor, giving the latter a free margin of a few millimeters.

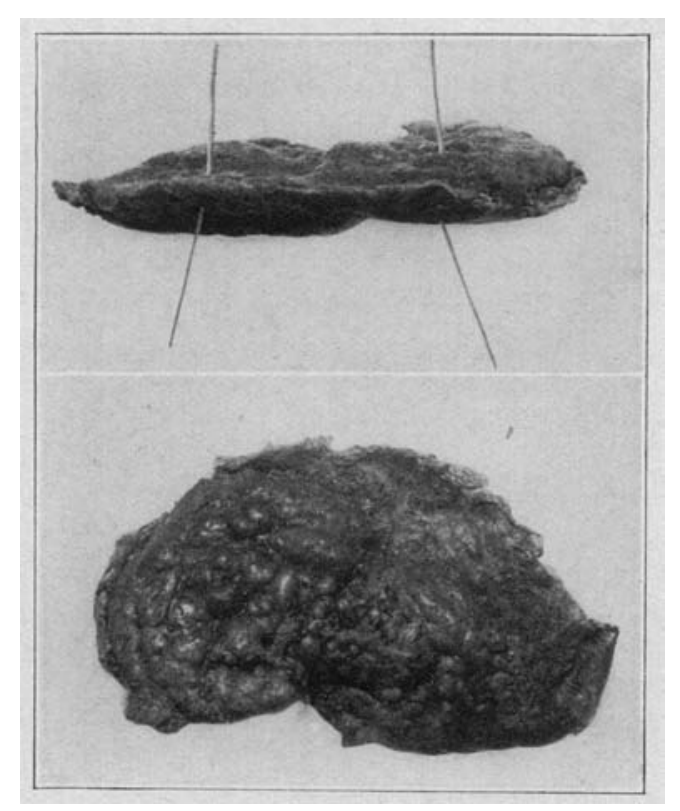

Figs. 6 and 7.-Photographs (natural size though considerably shrunken by fixation) of major portion of tumor; seen on edge in upper figure and from the inner dural surface in the lower figure.

It is quite possible that at the very depth of the sylvian cleft where the field became somewhat inaccessible, some portion of the involved bone and dura may have been left behind.

The dura was so vascular that silver clips were placed on its margins for purposes of hemostosis as the incision to outline the growth was being made. The situation and extent of the flat tumor is consequently well shown in the roentgen-ray picture subsequently taken (Fig. 3).

The operation, though a prolonged one, was well borne. Convalescence was umeventful (Fig. 4), and at the time of her discharge the exophthalmos and erlema of the orlit had largely disappeared.

At the present writing, ten months later, she remains free from symptoms; there is no indication of any tumor growth from the possible remaining fragment; the exophthalmos has almost completely subsided (Fig. 5). 
Pathologic Report.-The three chief fragments of tissue saved for study consisted of: (1) the primary tumor adherent to the under surface of the dura; (2) a portion of the thickened overlying skull; (3) a fragment of the temporal muscle.

1. The tumor proper. There are two fragments. The larger (Figs. 6 and 7) shows a flat growth with nodular surface, springing from the under side of a semicircular piece of dura measuring 5 by $3.5 \mathrm{~cm}$. in diameter. A margin of free dura surrounds the growth except at one portion of the specimen where the tumor has been cut into. At its thickest place the growth is only abou: $4 \mathrm{~mm}$. in thickness. On section it shows a typical endothelioma which is plastered on the inner surface of the membrane, the cell masses being arranged in fibrous alveoli (Fig. 8).

2. The bone fragment. (Figs. 9-11.) This is a wedge-shaped piece of bone measuring 5 by $3 \mathrm{~cm}$. in its surface diameters, and nearly $3 \mathrm{~cm}$. in its greatest thickness. The outer surface is roughened and irregularly grooved, and some fragments of muscle adhere to it. The inner surface, likewise, is rough and grooved, with fragments of firmly adherent dura. The third surface shows a cup of smooth eburnated bone evidently made by a large burr. A histologic study of the bone showed that its canals are everywhere invaded by tumor (Fig. 12).

3. The fragments of muscle removed early in the operation show on section (Fig. 13) invasion with tumor cells arranged in characteristic endothelial alveoli.

Dr. Victor Jacobson's pathologic note regarding the hyperostosis reads as follows :

"The bone is of cancellous type with the marrow spaces containing large groups of spindle cells arranged in the whorl-manner of dural endothelioma and similar to the tumor in the muscle described. The bone is dense and arranged in trabeculae. The resulting spaces contain the tumor cells which are surrounded by a thin compact layer of fibroblasts which in turn are separated from the bony trabeculae by loose connective tissue in which are a few thinwalled blood vessels. There is no apparent intravascular growth of tumor but the tumor cells have gained access to the marrow spaces and grown in them, often compressing the marrow vessels but not penetrating them. In one marrow space active hematopoiesis is in progress."

\section{INCIDENCE}

At the present writing (Jan. 1, 1922), in a series of considerably over one thousand presumptive cases of tumor, the lesion has been verified in 748 instances. ${ }^{1}$ Without going into the detail, unnecessary for our present purposes, of distinguishing between the various subvarieties of gliomas, adenomas and so on, these cases may be tabulated

1. The significance of the term "verified" and the way in which the brain tumor cases in the clinic are classified as verified, non-verified and tumorsuspects has been made clear in recent papers by Percival Bailey (Concerning the Clinical Classification of Intracranial Tumors, Arch. Neurol. \& Psychiat. 5:418-437 [April] 1921) and by C. E. Locke (A Review of a Year's Series of Intracranial Tumors, Arch. Surg. 3:560-581 [Nov.] 1921). 
as follows, in order to indicate the ratio of the endotheliomas to other main tumor groups :

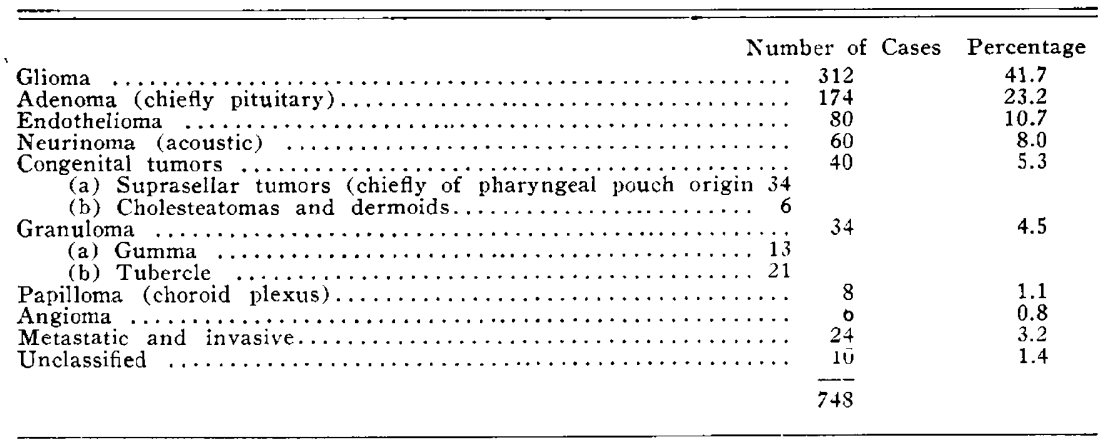

Thus, of the brain tumors encountered and histologically verified in this personal series, the endotheliomas represent 10.7 per cent. and occur in the ratio of one to four of the gliomas. ${ }^{2}$

\section{HYPEROSTOSIS CRANEI}

Of the eighty endotheliomas, twenty were accompanied by a recognizable thickening of the overlying bone. For reasons not as yet entirely clear, endotheliomas en plaque are more likely to provoke bony thickening from tumor invasion than are the large rounded tumors. Leaving out of consideration a few of the spreading endotheliomas of the basal meninges, such as those which arise from the gasserian envelops, ${ }^{3}$ nearly all of the flat tumors of the type recorded in the foregoing case report have produced an overlying hyperostosis. There have been eight of them in all, five in the anterior temporal region, as in the case recorded, usually with unilateral exophthalmos, and three in the parasagittal region of the vault.

The other twelve examples of hyperostosis have accompanied the rounded tumors which cause marked cerebral deformation. It was my early impression that these tumors were less likely to invade the bone because of their relatively small area of meningeal attachment. However, this cannot be the actual explanation, for the reason that there are several cases in the series in which a pronounced hyperostosis overlay an enucleable spherical endothelioma which proved to have a comparatively small area of meningeal attachment.

2. In his recent article on "The Accomplishments of Intracranial Surgery" (New York State J. M. 21:369, 1921), Dr. Frazier has made the statement that 60 per cent. of his cases were sarco-endotheliomas and only 38 per cent. gliomas.

3. Reference has already been made in another connection to the four endotheliomas in my series which have apparently arisen from the trigeminal sheath. "The Major Trigeminal Neuralgias," Am. J. M. Sc. 109:157, 1920. 


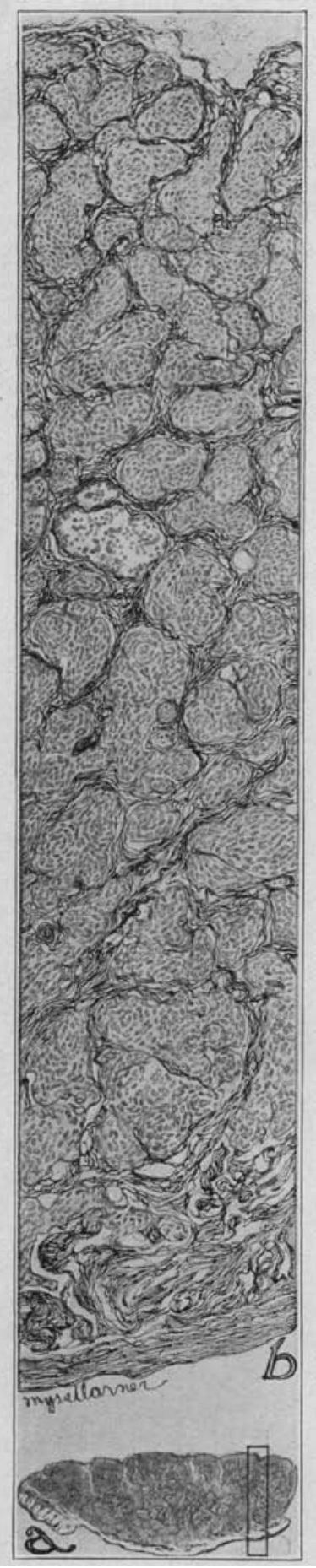

The endotheliomas must be regarded of course as benign tumors, but it is known that the growth tends to insinuate itself into crevices and may surround and incorporate important anatomic structures like the bloodvessels of the cranial base without damaging them. ${ }^{4}$ In view of this it might be assumed that under the influence of intracranial tension the tumor cells in the process of their multiplication become crowded into and through the vascular dural spaces and finally into the canaliculi of the bone. In consequence of this the bone becomes irritated, with subsequent osteoblastic proliferation which provokes the hyperostosis. There can be little doubt that the thickening occurs in this way, but intracranial tension can have nothing to do with it, in view of the fact that the flat endotheliomas which do not increase tension are, as we have seen, those which most often tend to invade the bone. There must be some other reason, therefore, to account for this peculiar process. One occasionally finds a meningeal endothelioma in which there is a tendency to bone formation within the tumor itself, and therefore the process may bear some relation to the bone-forming properties of the meninges, but without further data on the subject spectulation is futile. ${ }^{5}$

4. In another connection (with C. B. Walker) an example has been given of a basal endothelioma which had extruded itself far into the vaginal sheath of both optic nerves without destroying the tissue relationships. This case, one of the typical tumors arising from the olfactory groove, an example of which was pictured by Cruveilhier, was first reported in my monograph "The Pituitary Body and Its Disorders," Philadelphia, W. B. Saunders Co., 1912, and later on from its ophthalmological aspects with C. B. Walker: Arch. Ophth. 45:427 (Sept.) 1916, p. 427.

5. In an article with Lewis $\mathrm{H}$. Weed (loc cit.) we described and figured one of the endotheliomas of this series in which there were deposits of true bone, not only in the tumor but also in the arachnoid elsewhere.

Fig. 8.-Microscopic low-power drawing through entire thickness of tumor. 
The twenty examples of hyperostosis in the series of eighty endotheliomas represent one in four, but unquestionably this is too small a percentage if one takes into consideration the cases in which a slight thickening is observed on the inner surface of the sktull alone. In most

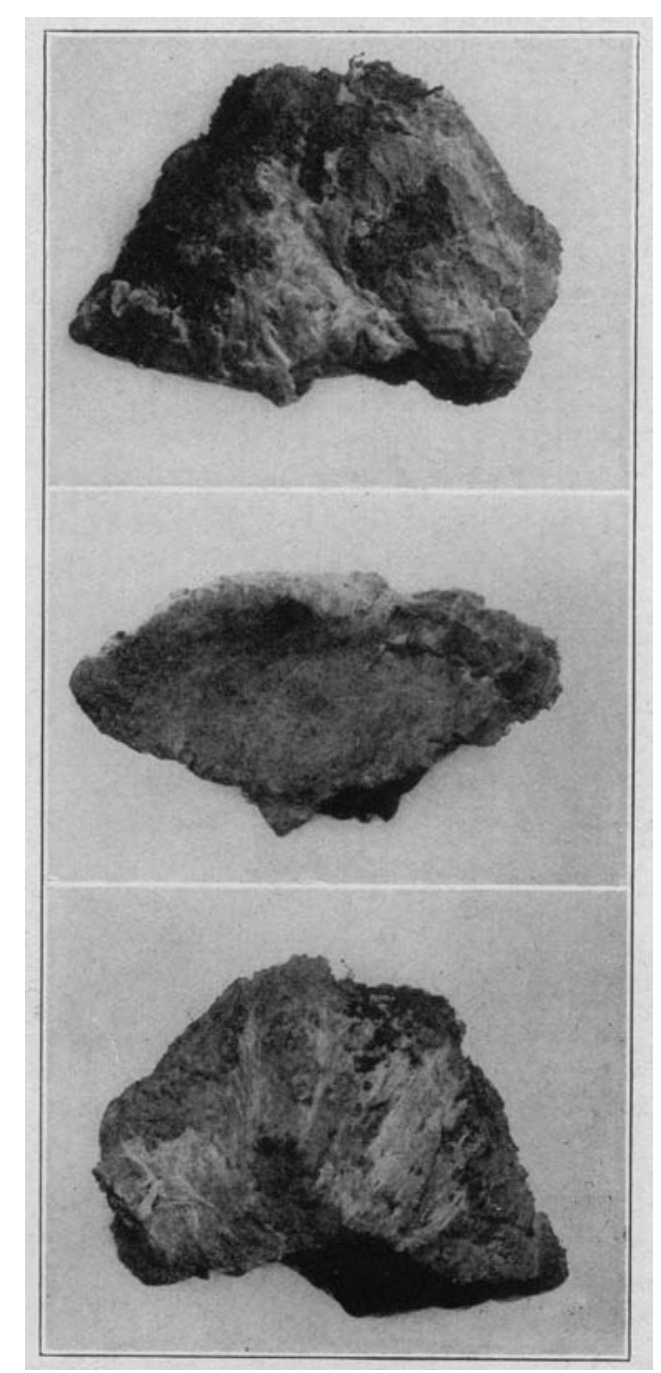

Figs. 9, 10 and 11.-Three views (natural size) of bone fragment, showing its outer surface in the upper figure, the dural surface in the lower figure, and its thickness in the middle figure. The dark points on the burred surface of the middle figure represent macroscopic evidences of tumor.

of the tumors of spherical type the point of origin of the growth from the meninges is clearly apparent. At this central core of the tumor the dura is apt to be indented by a slight endostosis, often too 
small to be recognized by the roentgen ray and in the operative notes of this long series of cases the partictlar fact may not always have been recorded.

In short, the tumors do not always behave alike in their effect on the bone. In some cases, aside from the increased vascularity with widening of the diploetic venous channels owing to stasis, there is no apparent change. In others, the bone may become greatly thinned

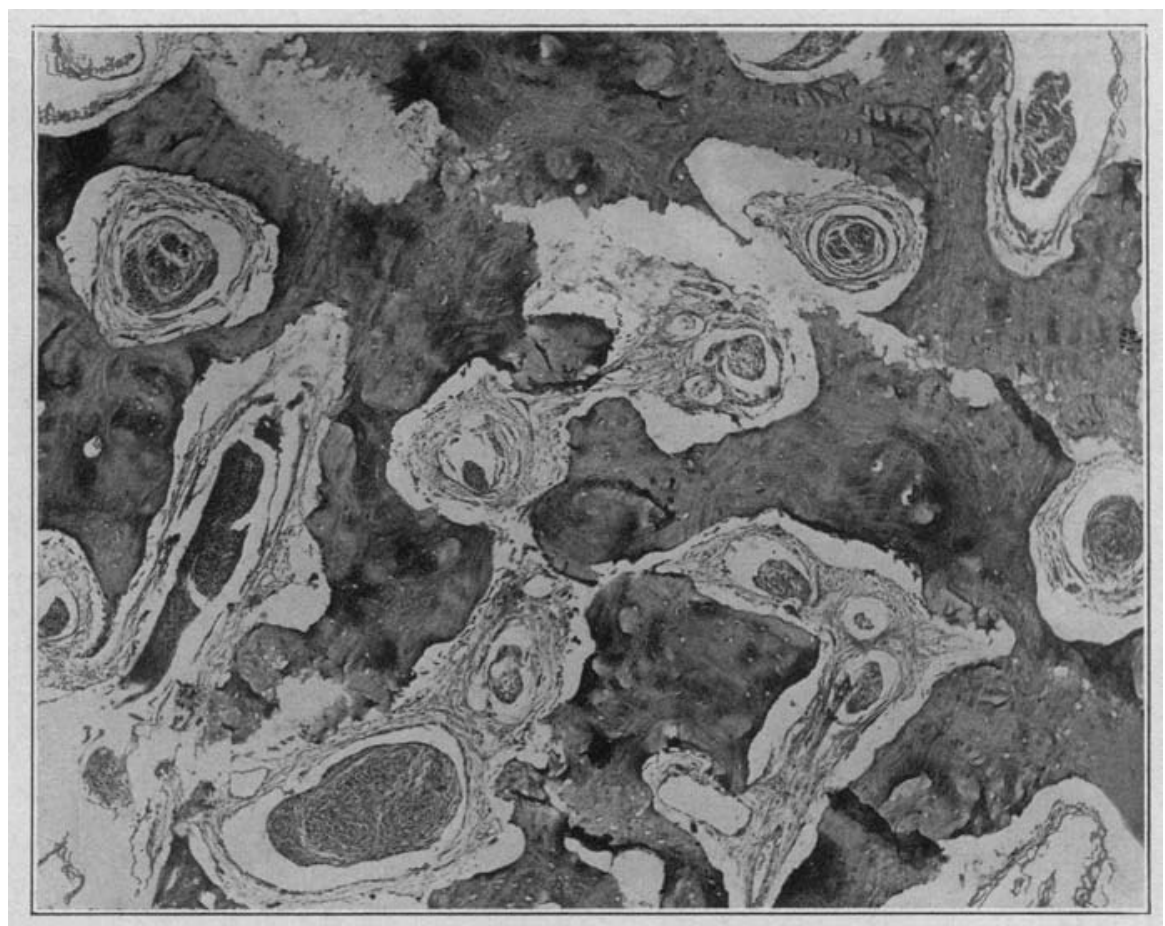

Fig. 12.-Low-power photomicrograph $(\times 60)$ of section of endethelial hyperostosis.

and the tumor find its way to the surface without any actual tumor penetration of clura. In still other cases, when a marked hyperostosis has been produced, the central portion of the hyperostosis may through subsequent absorption become occupied by tumor cells to the exclusion of anything more than occasional bony spicules, and in these cases the appearance of the bone in the roentgen ray may closely simulate a primary sarcoma of malignant character.

Apart from their relation to the cranial hyperostosis, these tumors have many features of unusual interest. Some of these points have been touched on in other connections. Thus, in a paper with Weed in our series of articles on the cerebrospinal fluid, the fact that they are in all probability of arachnoid rather than dural origin, as commonly 
believed, was touched on. It is our belief that they arise from the cell-clusters of the arahnoid villi projecting into the clura."

Cruveilhier's designation of these tumors, which he so well described, as "tumeurs cancérenses des meninges" means little more to us today than does the "fungous dura matris" of his predecessors; and as the term commonly employed, namely, dural endothelioma, gives the wrong impression of the membrane from which the growth has originated.

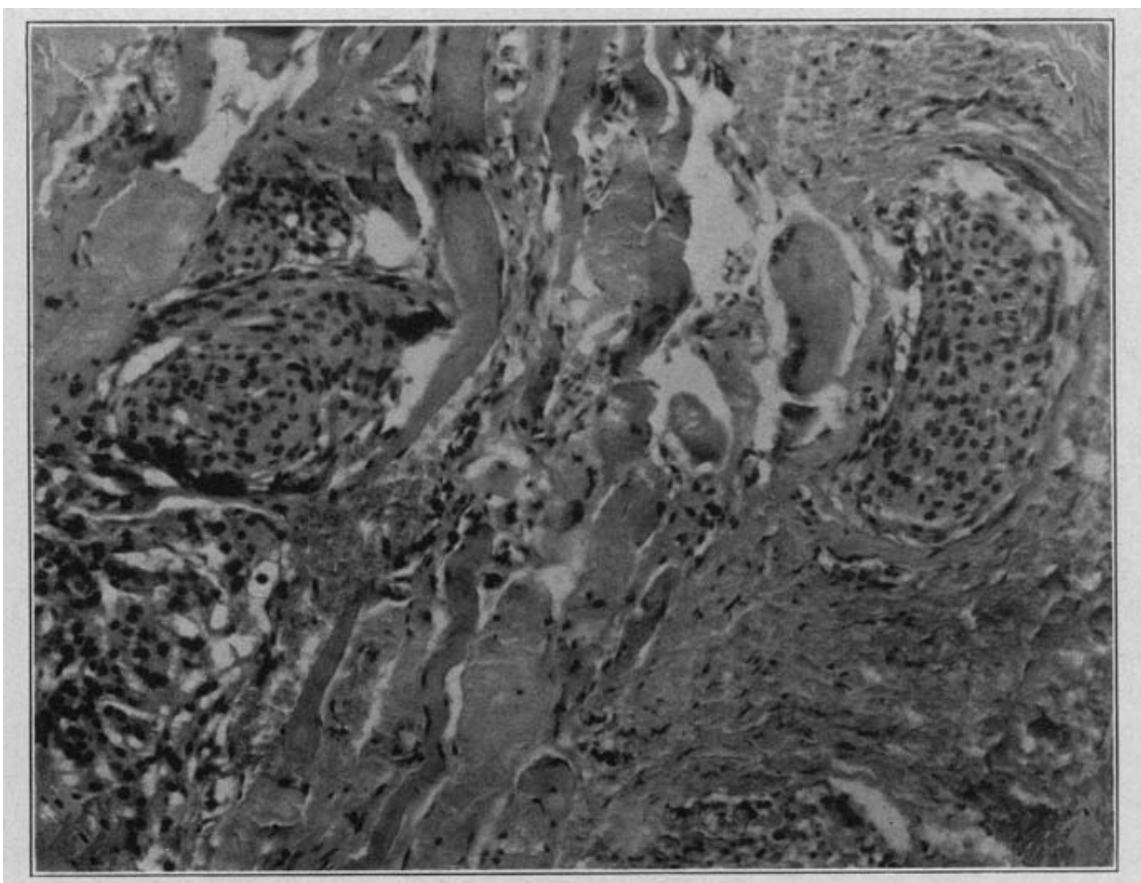

Fig. 13.-Section of temporal muscle $(X 170)$ showing extracranial endothelial invasion.

the designation meningotheliona or moningioma is suggested as a compromise. This at least avoids the objections which the embryologist might raise, for strictly speaking the growths would seem to be properly called mesotheliomas of arachnoid origin.;

6. Cushing. H., and Weed, L. H.: Studies of the Cerehrospinal Fluid and its Pathway. IX. Calcareous and Osseous Deposits in the Arachnoidea. Bull. Johns Hopkins Hosp. 26:367-372 (Nov) 1915.

7. There are other varieties of endotheliomas from which these common tumors must be distinguished, such as those of supposedly pial origin which have 110 diral attachment. These are exceedingly vascular growths, sometimes designated as hemangio-endotheliomas. Another type of tumor which is exceedingly rare in man but more common especially in the horse, is that form of endothelioma which arises from the choroid plexus. These tumors have been classified with the cholesteatomas, though the latter are of dermoid or epidermoid origin and bear no relation to the cndotheliomas. With endothelial tumors of these sorts we are not here concerned. 
Needless to say, were all these tumors associated with cranial hyperostoses they would be easily recognized and localized. Unfortunately they are not. Hence, in the absence of general pressure as well as of localizing symptoms, they may remain for a long time unrecognized and may ultimately reach such a size that their removal, even when they are accessible, is a most formidable operation. The largest tumor in the series weighed $246 \mathrm{gm}$., and was attended by extreme circulatory stasis. Even when focal symptoms do appear in the course of time, they may be of false localizing value, as was the case with one of these growths described in another connection.

The meningiomas, if they may be thus called, have certain. definite seats of predilection, and for the most part each group, on the basis of its anatomic seat of origin, gives a very definite and characteristic symptomatology. A discussion of these matters must await a more extended report on the subject. It is sufficient for this preliminary note to point out in summary:

1. That, in a series of over 700 verified tumors there have been eighty endotheliomas, their ratio to the gliomas being about one to four.

2. That at least 25 per cent. of the endotheliomas are accompanied by an overlying hyperostosis cranei which is either palpable externally or demonstrable by the roentgen ray. This process is due to invasion of the bony canals by tumor cells, with resultant stimulation of osteoblasts and the production of new bone.

\section{DISCUSSION}

Dr. William G. Spiller, Philadelphia: In $1899 \mathrm{my}$ attention was first called to the subject Dr. Cushing has discussed, and in that year Dr. Kirkbride and I reported a case of localized bony enlargement of the skull over a meningeal tumor before the Section on General Medicine of the College of Physicians of Philadelphia. I believe this was the first report of a case of this kind in literature. The condition was an endothelioma growing from the cerebral dura with endothelioma cells within the bony enlargement.

In 1903, Brissaud and Lereboullet reported two cases in which they considered the tumor of the brain as secondary to the enlargement of the bone of the skull. In one of these cases there had been an enlargement of the bone since childhood, and it was not until the age of 29 that symptoms of tumor developed. Multiple tumors were found growing from the dura. In 1907, I published a paper in The Journal of the American Medical Association containing in full a report of my first case, with the report of another similar case. In this second case the patient was syphilitic, and I believed that syphilis was the cause of the bony growth of the skull. A few other cases were reported before 1907 .

It has seemed important to recognize that where there is a localized large bony growth of the skull, tumor is likely to be found beneath or near it

8. Anosmia and Sellar Distortion as Misleading Signs in the Localization of a Cerebral Tumor, J. Nerv. \& Ment. Dis. 44:415-423 (Nov.) 1916. 
growing from the dura, and this fact is of great importance as regards operation. In one such case, with this thought in mind, I urged exploratory operation although the symptoms of intracranial tumor were not severe. The man refused operation and at necropsy a tumor growing from the cerebral dura was found.

I should like to ask Dr. Cushing in how many of his cases of meningeal tumor microscopic examination of the bony growth was made, and in what proportion of his cases he found by such microscopic examination tumor cells within the bony enlargement. I am willing to acknowledge that tumor cells may be found within the bony enlargement, and in my first case, reported in 1899 , such cells were found, but I have not been convinced that the bony enlargement in these cases is always secondary to the meningeal growth.

The matter is one of great importance. If we may believe that the bony enlargement is primary, we may hope to prevent the development of a meningeal tumor hy operation at an early date.

Dr. Charles A. Elsberg, New York: I have seen quite a number of cases with the bony changes of which Dr. Cushing has spoken; in two of the patients I had an experience concerning which I would like to ask Dr. Cushing. The involvement of the bone was so extreme that it would have meant an extensive removal of the cranium. In both I did not remove all the tumor containing bone. These patients have remained well, one for five and one for seven years, without further enlargement of the bone and without any symptoms, and apparently either the tumor in the bone grew very slowly or the bony growth was interfered with after part of the tumor had been removed.

Dr. Archibald Church, Chicago: The lack of symptoms in some of these cases is rather a startling clinical feature. I recall an elderly woman who developed a paraplegia with the symptoms of spinal cord tumor. Incidentally one dar, to calm her, I put my hand on her head and felt a large defect in the skull. A little further investigation revealed three openings in her skull, in any one of which a hen's egg might have been placed. These findings were confirmed by a roentgenogram of the skull. She never had had a head symptom nor had she complained about her head. At her death, three of these growths which had produced the large windows of the skull were found and also a somewhat similar growth causing spinal paraplegia.

In other cases affecting the brain and leading to operation, the symptoms had been comparatively insignificant.

Dr. Cushinc, in closing: In answer to Dr. Spiller's question, the reason why these conditions have not been fully studied is because there is a good deal of difficulty in sectioning bone; and when there is an obvious tumor, the histologic nature of which can be verified easily without decalcification, the overlying bone has been neglected.

In the preparation of this paper I have gone over all of the old specimens and practically all of them have been sectioned. I confess to a certain amount of hesitation. when the nature of a tumor has been verified, to subject an overhurdened department on pathology to the necessity of decalcifying the accompanying fragments of bone. However, tumor has been found in every case examined, and I judge that twenty or thirty have been gone over recently.

Dr. Elsberg's point is one with which I can fully concur. These growths are what Paget calls locally malignant tumors. The cases which $I$ have chosen for the lantern slide demonstration in connection with my paper have been the 
sphenotemporal tumors. On one slide half a dozen of them were shown. The bone, under those circumstances, is extensively involved throughout the whole temporal fossa The outer side of the orbit is involved, causing exophthalmos, and it is practically impossible in these circumstances to remove all of the affected bone. It is of course not difficult when the area of bony involvement is less inaccessible.

In reply to Dr. Church's question as to the lack of symptoms, the tumors may be multiple, but that is true also of the neurinomas, that is, the acusticus tumors, and there is a very interesting relation between these tumors and the meningiomas. My tumor series now includes sixty-seven acoustic tumors, all of them unilateral. When these lesions are single, there may be slight manifestations of a Recklinghausen's syndrome-a few fibromas on the skin, for example-but usually that is all. When, however, an acoustic tumor is bilateral, as they sometimes are, it is often accompanied by multiple meningeal tumors of the type under discussion. Dr. Bassoe and others have reported cases of this kind. Hence, there is some relation between tumors of these two types, but it is rare to find the lesions multiple. 